planning permission on grounds of over-provision. Individual homes are tending to become much larger, e.g. over 90 beds, and are increasingly passing from individual to corporate ownership. Financial return, rather than patients' need, could become a determining factor in a patients' management. Empty beds are financially undesirable. The admission of a patient to hospital or their transfer to a more suitable home could be disadvantageous to the current home when a high vacancy factor exists in the area.

When hundreds of dependent elderly are housed in adjacent buildings in areas chosen for available beds rather than personal associations, we are returning to the asylum village, not developing care in the community. We have institutionalised the community!

It has to be said that many of these new and large developments offer excellent care. Choice is desirable and competition should improve standards. Statutory services and the professions need to further clarify relationships with the independent sector as suggested in the College report mentioned above, to ensure the best service for elderly people is developed. NHS and Social Service provision in an area must be proportional to the numbers of dependent elderly not merely to the total elderly population.

Clatterbridge Hospital

M. T. MALCOLM

Bebington, Wirral

Merseyside L63 4JY

\section{Better use of out-patient and in-patient data in psychiatry: a necessary step towards medical audit}

DEAR SIRS

The continued failure to classify psychiatric beds into short, medium or long-stay makes audit and, therefore, effective planning impossible. Superficial scrutiny of one service in Scotland (West Lothian) shows, using nationally derived data, an average length of stay of 220 days which compares unfavourably with the Scottish average of 132 days.

More accurate information can be achieved when the beds are identified by classification. Hirsch (1988) showed for selected assessment units a mean length of stay of 38 days +13 . Those for the index hospital were 34.9 days in 1987. If this information was gathered nationally along with standard measures, such as percentage bed occupancy and turnover interval, medical audit is possible.

An internal audit could consider length of stay in specific diagnostic categories and relate these to specific clinical teams. In Scotland nationally first admission rates, while higher than in England, have been fairly consistent for over a decade. There has been a two-fold increase in readmission rates. Where there are "blocked" beds are rehabilitation services utilised at the correct time? Are current rehabilitation units dealing with an appropriate clientele?

A similar exercise would help in determining services for the elderly. For Scotland the national returns for the category geriatric assessment beds were a mean length of stay of 39.9 days with a turnover interval of 4.1 days. For the psychogeriatric assessment unit in West Lothian the mean length of stay was 52.7 days with a turnover interval of 4.7 days which compares favourably with the national average for Geriatric Assessment Units. Yet again national data classify all beds for the elderly as one in psychiatric hospitals.

Finally, and of greater significance in determining community-based services, the production of age specific information about new out-patients and the location of a clinic is highly relevant. New contacts in West Lothian were 1.32 per 100 population (Scottish average 0.74 ). When these were further broken down by age 15-65 and $65+$, new contacts for the index service were 1.30 and 2.34 respectively. McKechnie (1985) had already demonstrated that the rise in new psychiatric out-patients could be attributed to the development of out-patient facilities based in health centres.

Medical audit requires that the process should be relevant, objective, quantified, repeatable and able to effect appropriate change in the organisation of service and clinical practice Shaw \& Costain (1989). Simple classification if adopted nationally would make medical and, eventually, clinical audit attainable. Without such simple steps attention cannot be paid to resource management, audit, and especially, quality assurance.$$
\text { A. HaY }
$$$$
\text { A. A. MCKeChNIE }
$$$$
\text { Mental Welfare Commission for Scotland }
$$$$
25 \text { Drumsheugh Gardens }
$$$$
\text { Edinburgh EH3 } 7 R B
$$

\section{References}

HIRSCH, S. R. (1988) Psychiatric Beds and Resources: Factors influencing bed use and service planning. London: Gaskell (Royal College of Psychiatrists).

MCKeChNIE, A. A. (1985) The development of an integrated psychiatric service in a Scottish community. Acta Psychiatrica Scandinavica, 103, 72-97.

SHaw, C. D. \& CostaIN, D. W. (1989) Guidelines for medical audit: seven principles. British Medical Journal, 299, 4989.

\section{Standards of care revisited-medical audit in practice}

DeAR SIRS

The clinicians in the North Audit Group for Mental Handicap Psychiatry in the South West Region have 